\title{
Filtering Performance of Reducing the Sampling Rate of Sound Card: Perspectives on Different Signal-to-Noise Ratios
}

\author{
Bing-Yuh Lu ${ }^{* * *}$, Li-Man Tseng ${ }^{* * *}$, Ling-Yuan Hsu**, Shr-Hong Tang**, \\ Yu-Lin Weng ${ }^{* *}$, Man-Na Hsueh ${ }^{* * *}$, Mei-Ju Su ${ }^{* * * *}$, Huey-Dong $\mathrm{Wu}^{* * * * *}$, Jin-Shin Lai ${ }^{* * * * * *}$ \\ *Department of Electronic Engineering, Tungnan University, Taiwan, ROC \\ ${ }^{* *}$ Department of Information Management, Catholic St. Mary's Junior College of Medicine, Nursing, and Management, \\ Taiwan, ROC \\ ${ }^{* * *}$ Department of Nursing, Catholic St. Mary's Junior College of Medicine, Nursing, and Management, Taiwan, ROC \\ ${ }^{* * * *}$ Department of Biomedical Engineering, Yuanpei University, Taiwan, ROC \\ ${ }^{* * * * *}$ Section of Respiration Therapy, Department of Integrated Diagnostics and Therapeutics, National Taiwan University \\ Hospital, Taiwan, ROC \\ ****** Department of Physical Medicine and Rehabilitation, National Taiwan University Hospital and College of Medicine, \\ National Taiwan University, Taiwan, ROC
}

Benjaminee777@hotmail.com, liman@smc.edu.tw, lyhsu@smc.edu.tw, shrhongtang@smc.edu.tw, ylweng@smc.edu.tw, helena@smc.edu.tw, merri1024@gmail.com, hdwuntuh@ntu.edu.tw, jslai@ntu.edu.tw

\begin{abstract}
This study presented a theoretical analysis to prove that there exists an optimal sampling frequency to reduce the noise in the signal to reduce the processes of acoustic digital signal processing. The respiration sounds have been recorded by 2 to 44.1 $\mathrm{KHz}$ or higher sampling rates, however, the optimal frequency might be decided by the noise from environment. This study solves the problem from the point of theoretical view. The findings were interesting because the noise in a pure sine wave can be reduced by lower sampling rates. The sampling frequency of lung sound recording can be reduced in the range of 2 to $5 \mathrm{KHz}$. In addition, the results supported to enhance signal-to-noise ratio in the receivers of frequency modulation, under-water acoustics, and other communication applications.
\end{abstract}

Manuscript received Aug 20, 2013

This work was supported in part by the National Science Council, Taiwan under Grant 101-2221-E562-004, 101-2627-E-002-006, and 102-2627-E002-005.

B. Y. Lu is with the Tungnan University, Taipei, and St. Mary's Junior College of Medicine, Nursing and Management, Yilan, Taiwan. (e-mail: benjamin@smc.edu.tw)

L. M. Tseng is with St. Mary's Junior College of Medicine, Nursing and Management, Yilan, Taiwan. (e-mail: liman@smc.edu.tw)

L.Y. Hsu is with St. Mary's Junior College of Medicine, Nursing and Management, Yilan, Taiwan. (e-mail: helena@smc.edu.tw)

S.H. Tang with St. Mary's Junior College of Medicine, Nursing and Management, Yilan, Taiwan. (phone: +886-922-998305; fax: +886 2 39897398; e-mail: shrhongtang@smc.edu.tw)

Y. L. Weng is with St. Mary's Junior College of Medicine, Nursing and Management, Yilan, Taiwan. (e-mail: ylweng@smc.edu.tw)

M. N. Hsueh is with St. Mary's Junior College of Medicine, Nursing and Management, Yilan, Taiwan. (e-mail: helena@smc.edu.tw)

M. J. Su is with Yuanpei University,HsinChu, Taiwan (Email: merri1024@gmail.com)

H. D. Wu with Section of Respiration Therapy, Department of Integrated Diagnostics and Therapeutics, National Taiwan University Hospital, Taiwan (e-mail: hdwuntuh@ntu.edu.tw)

J. S. Lai with Health Science and Wellness Center, National Taiwan University, Taiwan (e-mail: jslai@ntu.edu.tw)
Keyword - noise reduction, sampling frequency, acoustic signal, lung sound, sound card, sonar,SNR.

\section{INTRODUCTION}

$\mathrm{T}$ HE linear interpolation technology for recovery of the signal is a common method, but the precision of the interpolation is not clear. In lung sound recording, the suggestive sampling rates are 44.1, 22.05, 11.025, 5.5125, and $2.75625 \mathrm{KHz}$ which are based on the sampling rate of traditional recorders. The sampling is the basic process of the signal processing. The modern lung sound and heart sound recorders, biomedical sensors, digital image systems, storage devices, digital communication systems get the acquisition of raw data from the humans by sampling $[1,2]$. The computing speeds of the micro-processors are enhanced by the semiconductor manufacturing technology which allowed the were $10 \mathrm{MHz} 30$ years ago, but that of the advanced communication signal converters are several GHz now [16].

The Computerized Respiratory Sound Analysis (CORSA) [3] pointed out the survey of respiratory sounds research, and very high speed internal clock to control the units of the processors. Therefore, the sampling rate of the electronic devices has been accelerated in the past decades. For example, the limitation of sampling rate of analog-to-digital converters the sampling rate was listed as a parameter of the method of digitalization [4]. The word lengths, and sampling rates were considered to record the lung sound perfectly by using the sound blaster cards or commercial multi-channel signal acquisition cards. The simplest process of the acquired raw data is moving average as a low pass filter to reduce the noise. The algorithm of adaptive filters acquired the environment noise to cancel the noise in the signals [5]. Furthermore, in the computation of the spectrogram, 
the overlapped data decided its resolution [6]. Therefore, the total error in a specific duration of lung sound can be a dominant factor of precision.

The adjustable sampling rate has been employed to reduce the volume in data storage and communication. Some literatures presented the adjustable sampling rate can enhance the signal-to-noise ratio [7,8]. In some cases, the weak signal with lower sampling rate, and the strong signal with higher sampling rate are the rules to reduce the data volume, and enhance the SNR. Therefore, the experimental results can be discussed by the theoretical analysis. The following proof proposed a direct solution to the optimal sampling rate for reducing the noise.

\section{METHOD}

If the signal $\mathrm{x}(\mathrm{t})$ with noise $\mathrm{n}(\mathrm{t})$, and the sampling rate is $\omega_{s}=\frac{2 \pi}{T}$, where $T$ is the period. The sampling pulse train is denoted as $p(t)$. Therefore, the sampled signal is:

$y_{p}(t)=(x(t)+n(t)) g p(t)$

where $p(t)=\sum_{n=-\infty}^{\infty} \delta(t-n T)$

In this study, the proof gives a solution to reduce noise by taking an optimal sampling frequency.

Taking the Fourier transform [9], we have:

$Y_{p}(j \omega)=(X(j \omega)+N(j \omega)) g P(j \omega)$

Generally, a low-pass filter with the transfer function of $H(j \omega)$ is always designed as be a pre-processing device to cancel the noise. Therefore, the noise can be optimal reduced by some specific sampling rate in the condition of

$\frac{d}{d \omega_{s}}(Y(j \omega)-X(j \omega) P(j \omega) H(j \omega))=0$

That is:

$\frac{d}{d \omega_{s}}\left(\frac{1}{T} \sum_{k=-\infty}^{\infty} N\left(j\left(\omega-k \omega_{s}\right)\right)\right)=0$

Then

$\frac{-j k}{T} \sum_{k=-\infty}^{\infty} N^{\prime}\left(j\left(\omega-k \omega_{s}\right)\right)=0$

The optimal $\omega_{\mathrm{s}}$ depends on the solution of the differential of $\sum_{k=-\infty}^{\infty} N^{\prime}\left(j\left(\omega-k \omega_{s}\right)\right)=0$. It exists the solution of $\omega_{s}$ to reduce the noise.

To verify the proof, we design a computer simulation to show the statistical view of the reduction of noise. Ideally, the respiration can be simplified as a sine wave whose half cycle for inspiration, and the other for expiration. The noise was the additive white Gaussian noise (AWGN) which is the common noise in the communication system [14]. The parameters of computer simulation were listed in Table 1.

The average absolute errors of the sampling points were computed to evaluate the performance of the method of reducing the sampling rate. The sampling rates were formulized as:

$$
f_{s i}=\frac{44.1}{2^{i-1}} K H z
$$

where $\mathrm{i}=1$ to 5 , are also the group indexes, i.e. the sampling frequency of group 1 was $44.1 \mathrm{KHz}$, and that of group 2 was $22.05 \mathrm{KHz}$.
The pure signal in group 1 was denoted as $\mathrm{x}_{1}[\mathrm{n}]$, and the noised signal was $\mathrm{y}_{1}[\mathrm{n}]$ whose numbers of points are $\mathrm{N}$. We reduce the sampling rate by dividing $2^{\mathrm{i}-1}$ where $i>1$. The numbers of samples were also reduced to be $\mathrm{N} / 2^{\mathrm{i}-1}$. However, the number of the samples can be recovered by well-known linear interpolation technology. Therefore,

$m e_{i}=\frac{\sum_{k=1}^{k=N}\left|\hat{y}_{i}-x_{1}\right|}{N}$

where $\hat{y}_{i}$ was the reduced noised signal and recovered by linear interpolation. Consequently, the percentage of error reduction $(\xi)$ can be described as:

$\xi(\%)=\frac{m e_{i}}{m e_{1}} \cdot 100 \%$

\section{RESULTS}

The ideally pure sine wave and that with the AWGN and random noise $(\mathrm{RN})$ of signal to noise ratio $(\mathrm{SNR})=40 \mathrm{~dB}$ were shown in Fig. 1 (a) and (b). The sampling rate was $44.1 \mathrm{KHz}$. The root-mean-square (RMS) error was computed by different sampling rates of 44.1(Group 1), 22.05(Group 2), 11.025(Group 3), 5.5125(Group 4), and 2.76125(Group 5) $\mathrm{KHz}$, respectively. We run 100 times of computer simulations by the same parameters in Table 1 to compute the mean absolute errors (MAE). The box plots were presented in Fig. 2.

TABLE I THE PARAMETERS of the SIMULATED RESPIRATION SIGNAL.

\begin{tabular}{ll}
\hline \hline \multicolumn{1}{c}{ Variables } & \multicolumn{1}{c}{ Value } \\
\hline Shape & Sine wave \\
Period & $3 \mathrm{sec}$. \\
Signal to noise ratio & 20,30, 40, and 50 dB \\
(SNR) of AWGN & \\
Sampling rate & 44.1(Group 1), 22.05(Group 2), 11.025(Group \\
& 3), 5.5125(Group 4), and 2.76125(Group5) \\
& $\mathrm{KHz}$ \\
\hline
\end{tabular}

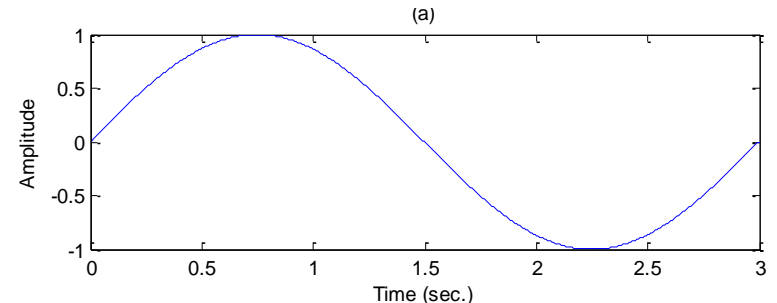

(b)

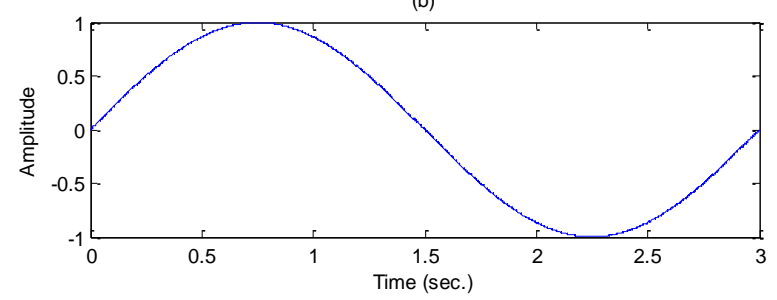

Fig. 1. Computer simulation: (a) the ideally pure sine wave and (b) that with AWGN. 
(a)

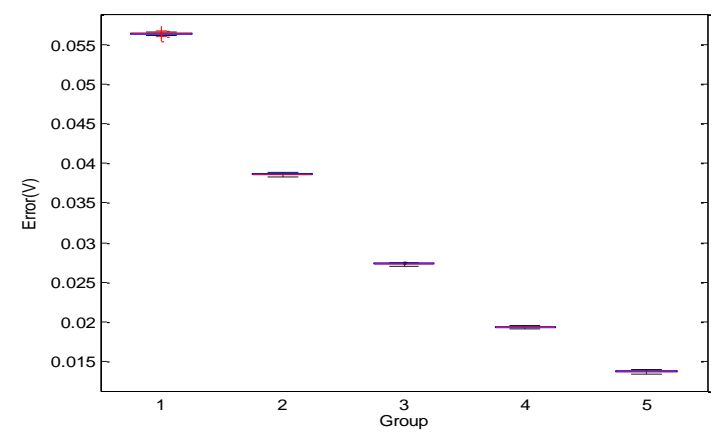

(b)

$30 \mathrm{~dB}$

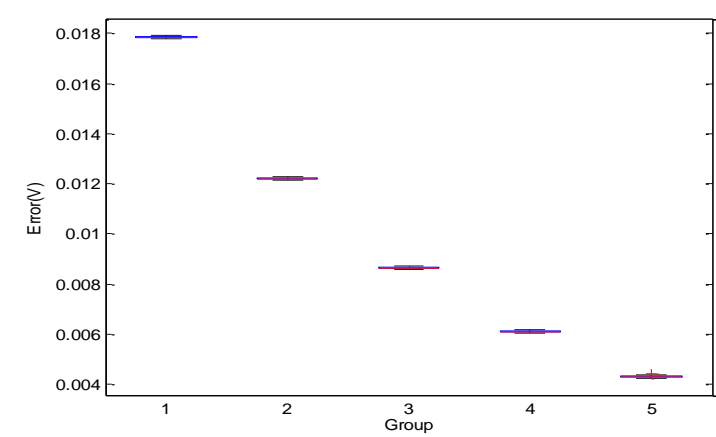

(c)

$40 \mathrm{~dB}$

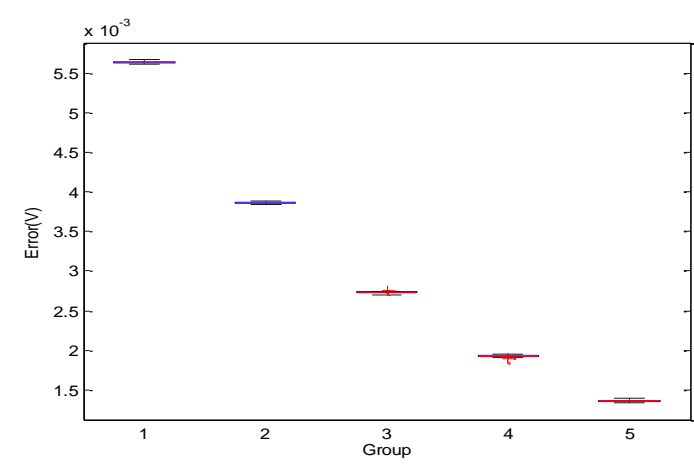

(d)

$50 \mathrm{~dB}$

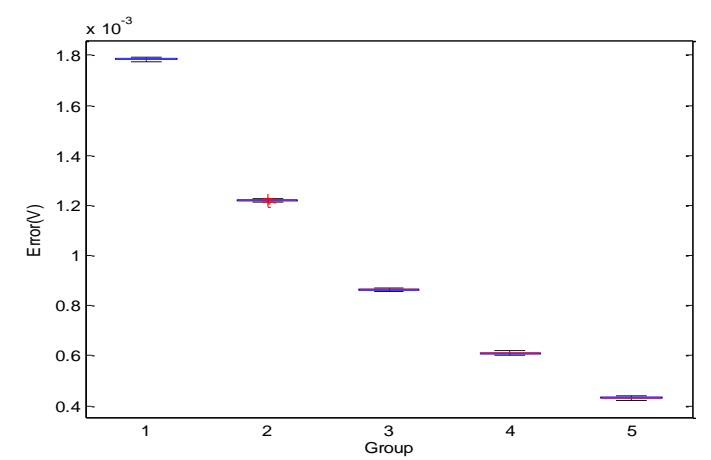

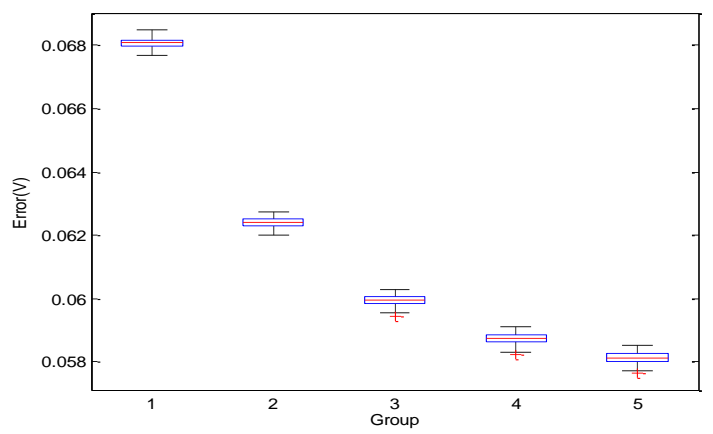
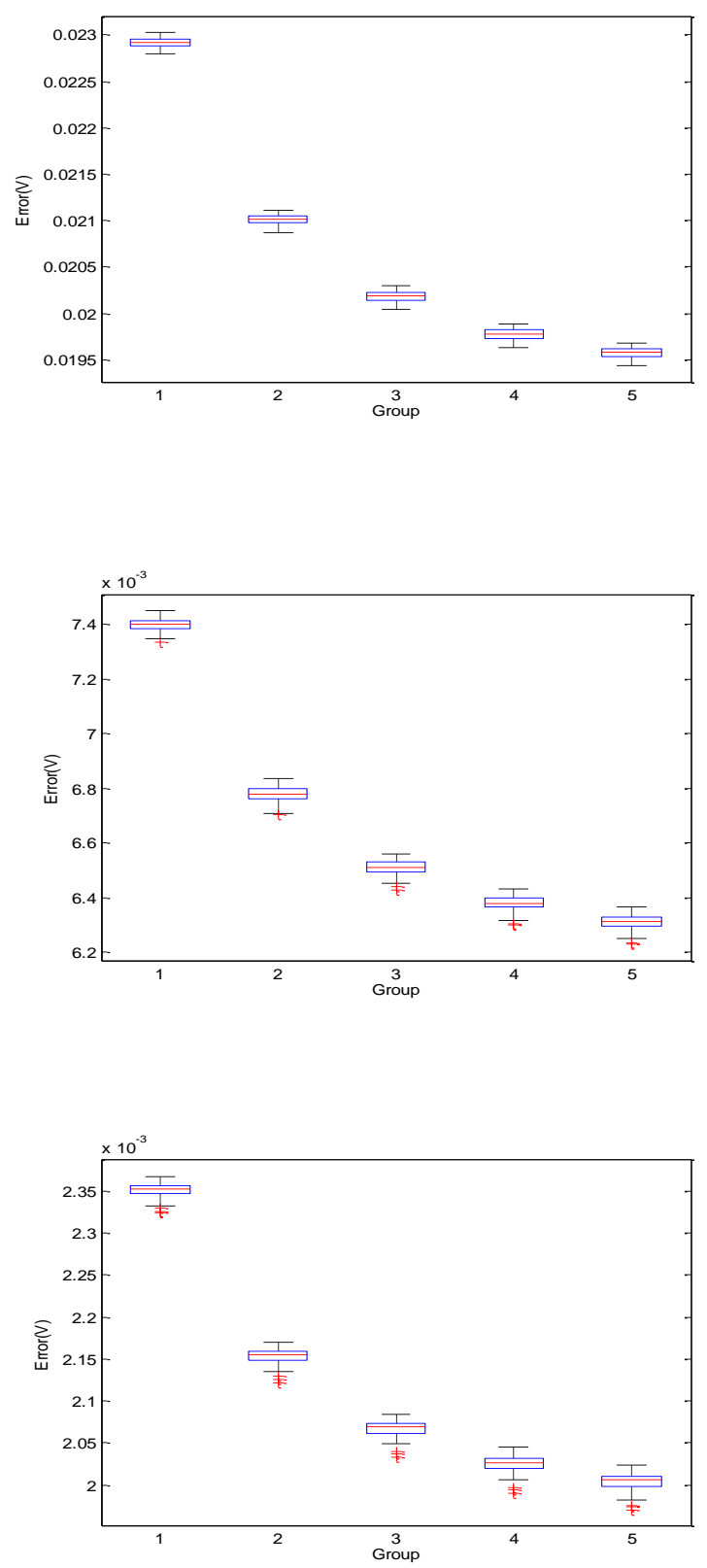

Fig. 2. The box plots of the MAE of Groups 1, 2, 3, 4, and 5 of 100 times. The AWGN level were (a) SNR=20, (b) SNR=30, (c) SNR=40, and (d) SNR=50. (The sampling rates: 44.1(Group 1), 22.05(Group 2), 11.025(Group 3), 5.5125(Group 4), and 2.76125(Group 5) KHz) 
(a) $20 \mathrm{~dB}$

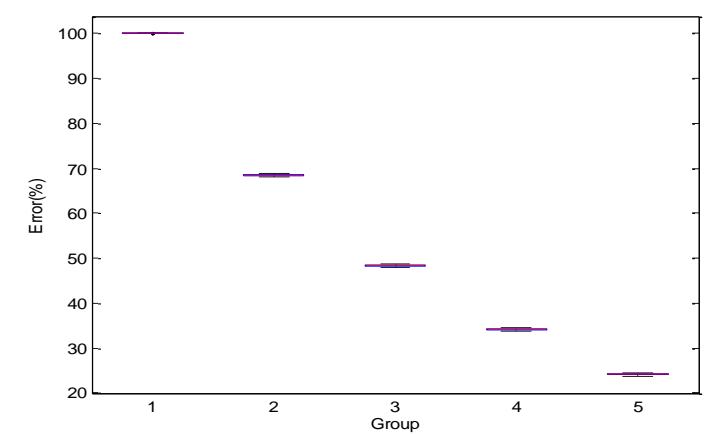

(b)

$30 \mathrm{~dB}$

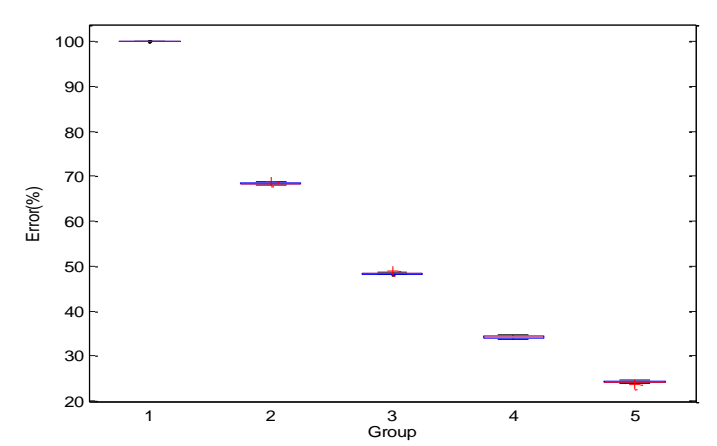

(c)

$40 \mathrm{~dB}$

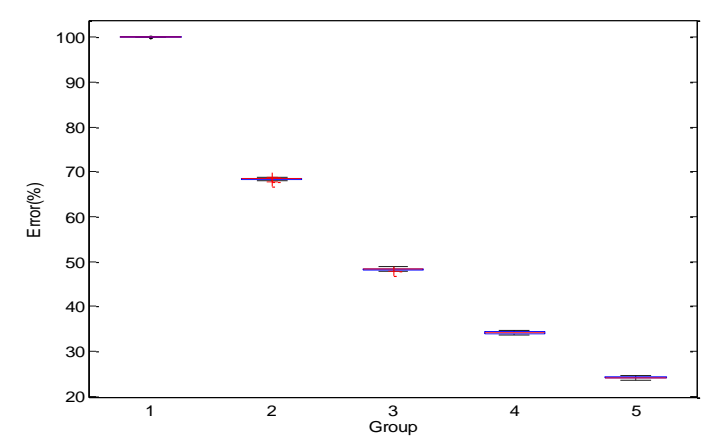

(d)

$50 \mathrm{~dB}$

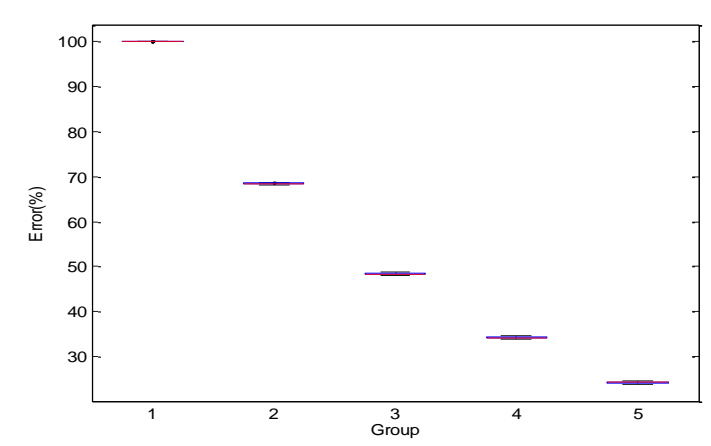

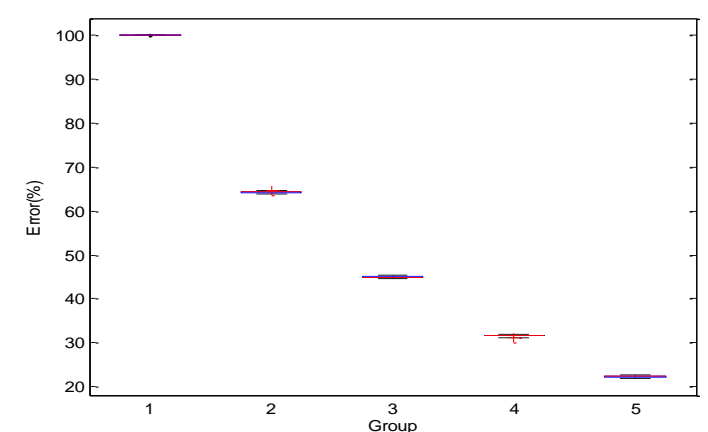
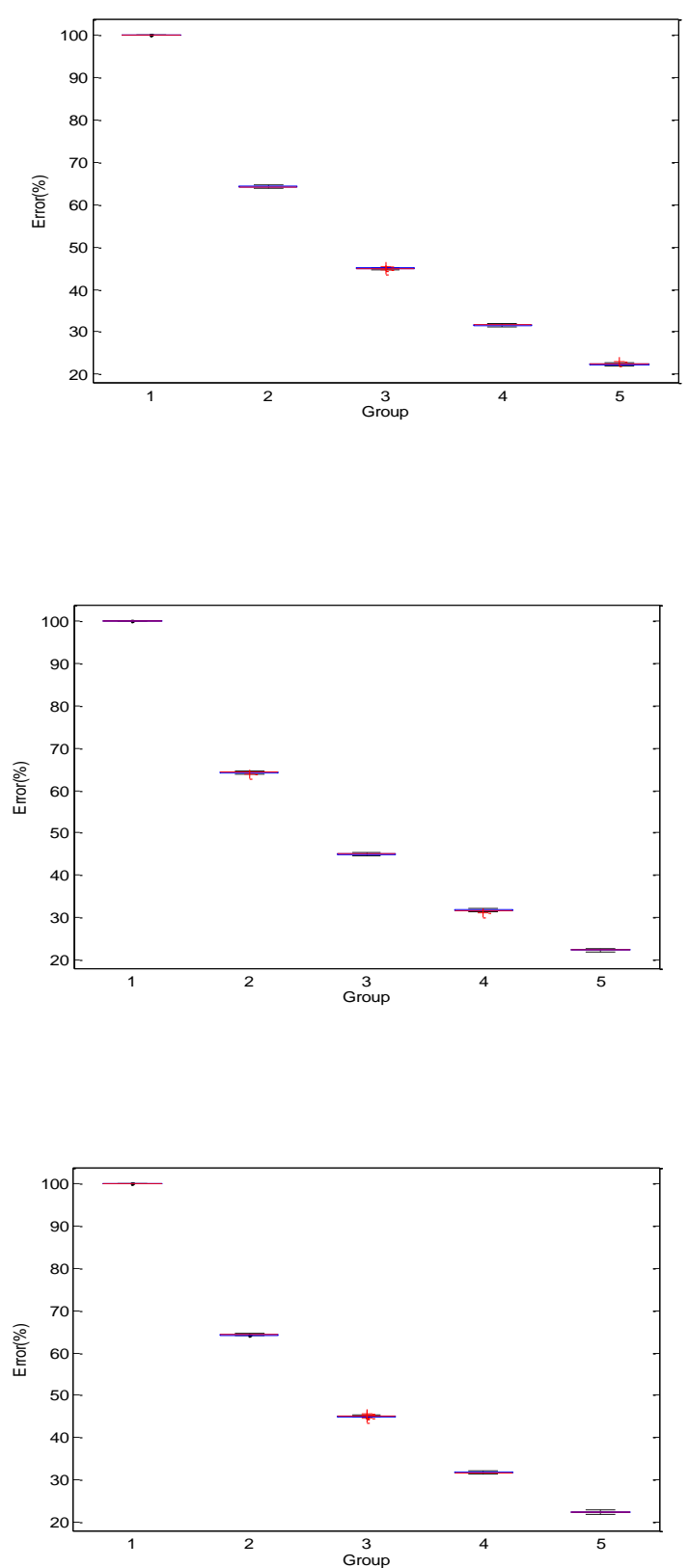

Fig. 3. The box plots of the percentage of error reduction ( $\xi$ ) of Groups 1, 2, 3, 4, and 5 of 100 times. The AWGN level were (a) SNR=20, (b) SNR=30, (c) $\mathrm{SNR}=40$, and (d) SNR=50. (The sampling rates: 44.1(Group 1), 22.05(Group 2), 11.025(Group 3), 5.5125(Group 4), and 2.76125(Group 5) KHz)B 
Figure 2 presented the statistical results. Each box contained 100 trials of MAE. In (a), the SNR of AWGN is $20 \mathrm{~dB}$. The box plots displayed that Group 5 ( sampling rate $=2.76125 \mathrm{KHz}$ ) has gotten the minimum MAE errors. Compared with the original signal (Group 1), the operation of reducing sampling rate cancelled about 1/5 MAE errors from the point of statistical view. In (b), (c), and (d), the SNRs were 30, 40, and $50 \mathrm{~dB}$. All results show that the smallest sampling rate (Group 5) gets the smallest RMS errors. Based on the proof, there exists a optimal sampling rate to reduce the noise. From the box plots, we find that the lower sampling rate got the lower MAE errors by statistical analysis.

The percentage of error reduction by decreasing the sampling rate was obviously. Figure 3 presented the performance of the reducing sampling frequency. All plots displayed that group 5 achieved the best effect of noise cancellation for both AWGN and RN. The $\xi$ s were in the range of 15 to $25 \%$. Therefore, the filtering performance of the easy method for pure sine waves was excellent.

From the observation of Figs. 2, and 3, the lower sampling rate did reduce both AWGN and RN. The lower frequency signal can be filtered the noises by the lower sampling rate of sound card.

\section{DISCUSSION}

The pure sine waves usually play as the carrier in the communication systems [17]. In the detection systems, the reflections of the transmitting pure sine waves are widely employed in sonar, RADAR, and other systems. The sampling rate of receiver can be reduced based on the results of our study, especially in the real-time computing system. Fourier series described that a periodic signal is the linear combination of the fundamental signal and its harmonics. Therefore, the simulated ideally pure sine wave is significant, because of the theory of the linear time invariant (LTI) system [1]. The box plots can be expanded to explain the geniality of the operation of the reducing sampling rate. Chang and Lai [11] have studied on the performance evaluation and enhancement of lung sound recognition system in two real noisy environments. The results presented that the frequencies of noises were higher than that the main episodes in the spectrogram. Therefore, if the sampling rate of the experiment reduced to a specific frequency and processed the raw data with moving average, most of the environmental noise can be much cancelled. However, the experienced medical staff usually records the lung in silent environments whose noises compose with the sounds from air conditioners, fans, computer systems and so on. Based on the sampling theory, the energy of noise would be reduced by the lower sampling rate that shows the possibility of the optimal sampling rate can be found in some specific environments. Furthermore, the processing of the digital data can be accelerated by decreasing the data. The benefits of selecting a better sampling rate can achieve the better performance of signal processing. CORSA indicated that the wheeze was contained in the domain frequency at $400 \mathrm{~Hz}$, but a number of investigators have suggested that the range is actually between $80-1,600 \mathrm{~Hz}$ and $350-950 \mathrm{~Hz}$ by filter theory $[12,13]$. Lu et al [14] have synthesized the normal breath and wheezing sounds whose main components were under $2 \mathrm{KHz}$. All the papers provided that the evidence of the sampling rate can be reduced to cancel the noise. However, the crackle includes the higher frequency components, the optimal sampling rate can be higher than normal breath and wheezing sounds. This is the limitation of using the lower sampling rate for lung sound recording. Furthermore, the standardized sampling rates applied in many industry standard sound facilities are 44.1, 22.05, 11.025 or $5.5125 \mathrm{KHz}$ as standards. Most modern research groups selected 44.1 or $22.05 \mathrm{KHz}$ to record the lung sound. The filtering performances of AWGN and $\mathrm{RN}$ were close. Therefore, the filtering method can be employed in the receivers of digital systems to cancel the noises caused from different reasons.

Some electronic stethoscope use the lower frequency of sampling rate [18]. It supports the examination of this study. We give the better choice of sampling rates to the researchers of respiration sounds.

\section{CONCLUSION}

The theoretical analysis of reducing sampling rate filter was solved in this study. The proof can be employed to simplify the filter design, if we get the smallest error from the simulated signal by the optimal sampling frequency. In addition the reasons of noises in physiologic signals are usually bandlimited, therefore, the optimal sampling frequency can be used to simplify the system design. In the detection systems, the reflections of the transmitting pure sine waves are widely employed in sonar, RADAR, and other systems. The sampling rate of receiver can be reduced based on the results of our study, especially in the real-time computing system.

\section{ACKNOWLEDGEMENTS}

The authors thank for the valuable comments from the reviewers and the supports from the project numbers of NSC101-2221-E-562-004, NSC101-2627-E-002-006, and NSC102-2627-E-002-005, National Science Council, Taiwan, Republic of China.

\section{REFERENCES}

[1] S. Haykin and B. Van Veen, Signals and systems, New York: Wiley, 1998. [2] J. S. Beasley and G.M. Miller, Modern electronic communication. New York: Pearson Education, 2005.

[3] A. R. A. Sovijarvi, J. Vanderschoot and J. R. Eavis, Standard of computerized respiratory sound analysis. Eur Respir Rev 2000;10:585.

[4] J. E. Earis and B. M. G. Cheetham, "Current methods used for computerized respiratory,” Eur Respir Rev, vol. 10, 2000 pp. 586-590.

[5] B. S. Lin, H. D. Wu, F. C. Chong and S. J. Chen, "Wheeze recognition based on 2D bilateral filtering of spectrogram," Biomedical EngineeringApplications, Basis \& Communications, vol 18, 2006, pp. 128-137.

[6] A. R. A. Sovijärvi, L. P. Malmberg, G. Charbonneau, J. Vanderschoot, F. Dalmasso, C. Sacco, M. Rossi and J. E. Earis. "Characteristics of breath sounds and adventitious respiratory sounds," Eur Respir Rev, vol. 10, 2000, pp. 591596.

[7] J. P. McKay and Y. Rahmat-Samii, "Compact range reflector analysis using the plane wave spectrum approach with an adjustable sampling rate," IEEE Trans. on Ant and Propaga, vol. 39, 1991, pp.746-753.

[8] A. Hart and S. P. Voinigescu, "A $1 \mathrm{GHz}$ bandwidth low-pass $\Delta \Sigma$ ADC with $20 \mathrm{GHz}$ to $50 \mathrm{GHz}$ adjustable sampling rate," IEEE Radio Frequency Integrated Circuits Symposium, vol. 2, 2008, pp. 181-184.

[9] F. G. Stremler, Communication systems, New York: Pearson Education, 2005 . 
[10] L. Vannuccini, J. E. Earis, P. Helistö, B. M. G. Cheetham, M. Rossi, A. R. A. Sovijärvi and J. Vanderschoot, "Capturing and preprocessing of respiratory sounds," Eur Respir Rev, vol. 10, 2000, pp. 616-620.

[11] G. C. Chang and Y. F. Lai, "Performance evaluation and enhancement of lung sound recognition system in two real noisy environments," Computer Methods and Programs in Biomedicine, vol. 97, 2010, pp. 141-150.

[12] N. Gavriely, Y. Palti, G. Alroy and J. B. Grotberg. "Measurement and theory of wheezing breath sounds," J Appl Physiol, vol 57, 1984, pp. 481-492. [13] H. Pasterkamp, A. Tal, F. Leahy, R. Fenton and V. Chernick. "The effect of anticholinergic treatment on postexertional wheezing in asthma studied by phonopneumography and spirometry," Am Rev Respir Dis, vol. 132, 1985, pp. $16-21$

[14] B. Y. Lu, H. D. Wu, S. R. Shih, F. C. Chong, M. L. Hsueh and Y. L. Chen, "Combination of frequency and amplitude modulated Model for the synthesis of normal and wheezing sounds," Austra Phy Eng Sci Med, vol.34, 2011, pp. 449-457.

[15] V. M. Rossi, A. R. A. Sovijärvi, P. Piirilä, L. Vannuccini, F. Dalmasso and J. Vanderschoot, "Environmental and subject conditions and breathing manoeuvres for respiratory sound recording," Eur Respir Rev, vol. 10, 2000, pp. 611-615.

[16] PICO 900 Series Scope. Available:

http://www.picotech.com/picoscope9000.html

[17] T. Wheel, Electronic communication systems for technicians, New

York: Pearson Education, 2006.

[18] User manual, 3M Littman electronic stethoscope, Model 3200.

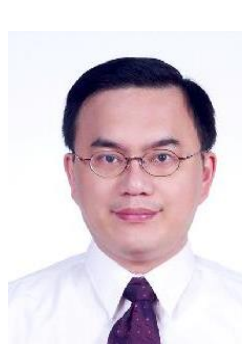

Bing-Yuh Benjamin Lu received his BS degree in electrical engineering from National Central University and his MS and $\mathrm{PhD}$ degrees in electrical engineering from National Taiwan University in 1988, 1993, and 2000, respectively.

He was an instructor at the Department of Electronic Engineering, Tungnan University, New Taipei City, Taiwan, Republic of China in 1992. In 2000, he became an associate professor in the same department. He joined the Department of Information Management, St. Mary's Medicine, Nursing, and Management College, Yi-Lan, Taiwan, Republic of China in 2011, and served as an associate professor and the department head in the duration of 2011 to 2013. He is currently an associate professor in Department of Electronic Engineering, Tungnan University, New Taipei City, Taiwan, Republic of China. He is interested in computer simulation, medical engineering, acoustics, lung sound and educational researches. $\mathrm{He}$ is a member of IEEE.

His academic interests in focus on acoustics, medical engineering, computer simulation, educational applications of engineering, and pulmonary signal processing especially about lung sound and pulmonary mechanism.

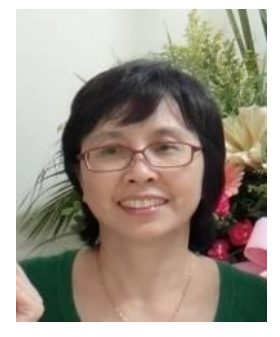

Liman Tseng is a lecturer in the Nursing Department, St. Mary's Junior College of Medicine, Nursing and Management, YiLan, Taiwan. She has a MS degree in nursing, Tzu Chi University in 2005, who attended a $\mathrm{PhD}$ degree in public health, in Tzu Chi University in 2009. Her research and teaching interests include nursing administration, surgical nursing, oncology nursing and pediatrics nursing.

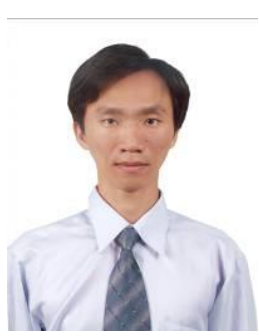

Ling-Yuan Hsu was born in I-Lan, Taiwan, R.O.C., in 1975. He received the M.S. degree in computer science and information engineering from National Dong Hwa University, Hualien, Taiwan, in 2004, and Ph.D. degree in computer science and information engineering at National Taiwan University of Science and Technology, Taipei, Taiwan, in 2013. Currently, he is an assistant professor in the Department of Information Management of ST. Mary's Medicine, Nursing, and Management College. His research interests include artificial intelligence, image processing, evolutionary computation, and computer networks.

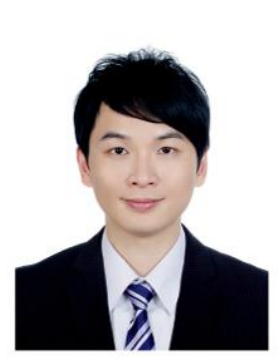

Shr-Hong Tang was born in Taiwan in 1978. He received Ph.D. in Engineering from National Chiao Tung University (Hsin-Chu, Taiwan) in 2007. His major field of study was applied information technology in engineering. $\mathrm{He}$ is currently an assistant profession in St. Mary's Medicine, Nursing and Management College, and pursuing an advanced degree in computer science from National Taipei University of Education (Taipei, Taiwan)

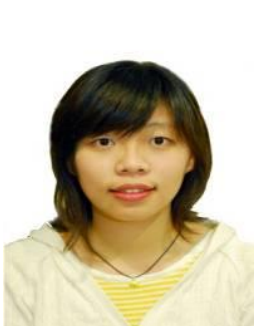

Yu-Lin Weng received her M.S. degree in Information Management from National Taichung University of Science and Technology and her MS degrees in Network Learning technology from National Central University in 2006 and 2008, respectively. Currently, she is pursuing a $\mathrm{PhD}$ degree in Information Technology at National Taiwan University of Science and Technology .She was an instructor at the Department of Information Technology, St. Mary's Medicine, Nursing and Management College, Yi-Lan, Taiwan, Republic of China from 2008. She is currently the head of the Registration Section, St. Mary's Medicine, Nursing, and Management College. She is interested in digital learning, adaptive testing, and database systems.

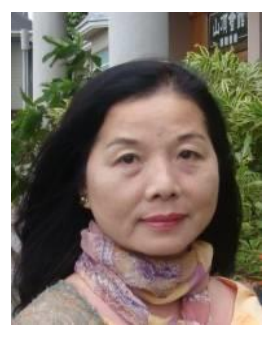

Man-Na Hsueh received her MS degree in nursing from National Taipei University of Nursing and Health Sciences in 2004. Over the past 35 years, she has been teaching at St. Mary's Nursing School (before 2005) and St. Mary's Medicine, Nursing, and Management (after 2005). She was the Director of the Department of Students' Affairs from 1991 to 
1997, the Director of the Department of Students' Internship Affairs from 2004 to 2005, and the Representative President and Director of the Department of Academic Affairs in 2005. She is currently the head of Department of Nursing at St. Mary's Junior College of Medicine, Nursing, and Management . She is interested in elder person's health care and in community medicine.

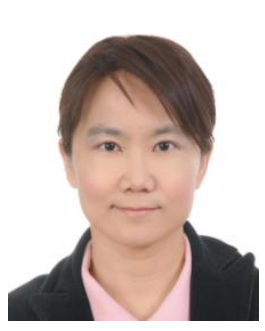

Mei-Ju Su received Ph.D. degree at Graduate Institute of Electronics Engineering, National Taiwan University since 2004 to 2010, Master degree at Department of Electrical Engineering, Syracuse University since 1996 tp 1997. Now, her researches focus on biosignal processing, medical device, telehealthcare system, and sensor design. She had been worked at Industry for 8 years since 1998 2005 on network IC design and SiS Company, medical instrument system design at Biomedical Technology and Device Research Laboratories of Industrial Technology Research Institute. Now she is the associate professor of department of Biomedical engineering, Yuanpei University.

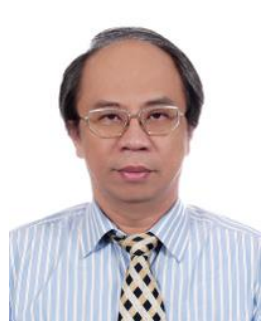

Huey-Dong Wu was graduated with a M.D. degree from Medicine Department of National Taiwan University in 1984 . He received resident training in National Taiwan University Hospital (NTUH) and trained as visiting scholar in ULCA later. He service in the Department of Internal Medicine and Department of Integrated Diagnostic \& Therapeutics in National Taiwan University Hospital (NTUH) till now. He is the chief of the division of Respiratory Care, Department of Integrated Diagnostic \& Therapeutics in NTUH. His academic interests in focus on respiratory care, pulmonary pathophysiology and pulmonary signal processing especially about lung sound and pulmonary mechanism.

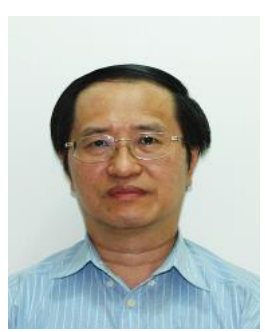

Jin-Shin Lai was born in Taipei, Taiwan, Republic of China (R.O.C.) in 1949. He received the M.D. degree from the Medical School of National Taiwan University, Taipei, Taiwan in 1974. He completed residency training in the Department of Physical Medicine and Rehabilitation, National Taiwan University Hospital, Taiwan in 1978.He was a Lecturer, Associate Professor, and Professor of Medical School, College of Medicine, National Taiwan University in 1980, 1984 and 1996, respectively.

Now, he is the Director of Health Science and Wellness Center, National Taiwan University and Director of Sports Medicine Center, National Taiwan University Hospital. His professional interests include medical informatics, sports medicine, rehabilitation medicine, biomechanics, and rehabilitation engineering. He and his research group had published a series of studies about Physiological Effects of Tai Chi Chuan Training.

Prof. Lai has been the Chairman of School of Rehabilitation Medicine (1988-1992), Chairman of School of Occupational Therapy (1992-1998), Chairman of Department of Physical Medicine \& Rehabilitation (1993-1999), Chief of Division of Medical Informatics (2001-2007), College of Medicine, National Taiwan University, respectively. He also served as the Chairman of Department of Information Technology \& Service, National Taiwan University Hospital (1998-2004), and elected as the President of Rehabilitation Medicine Association of Taiwan (R.O.C.) 1993-1999, the President of Sports Medicine Association of Taiwan (R.O.C.) 1997-2001, the Chairman of Scientific Commission of Asian Federation of Sports Medicine (AFSM) 1990-2000, the Treasurer of Asian Federation of Sports Medicine (AFSM) 2001-2004 and the Chair of HL-7 Taiwan (2005-2009). 